\title{
WHY IS THE CONCLUSION OF THE GERDA EXPERIMENT NOT JUSTIFIED?
}

\author{
H.V. Klapdor-Kleingrothaus ${ }^{*}$ and I.V. Krivosheina ${ }^{\dagger}$ \\ Heidelberg, Germany
}

October 29, 2018

\begin{abstract}
The first results of the GERDA double beta experiment in Gran Sasso were recently presented. They are fully consistent with the HEIDELBERG-MOSCOW experiment, but because of its low statistics cannot proof anything at this moment. It is no surprise that the statistics is still far from being able to test the signal claimed by the HEIDELBERG-MOSCOW experiment. The energy resolution of the coaxial detectors is a factor of 1.5 worse than in the HEIDELBERG-MOSCOW experiment. The original goal of background reduction to $10^{-2}$ counts $/ \mathrm{kg} \mathrm{y} \mathrm{keV}$, or by an order of magnitude compared to the HEIDELBERG-MOSCOW experiment, has not been reached. The background is only a factor 2.3 lower if we refer it to the experimental line width, i.e. in units counts/kg y energy resolution.
\end{abstract}

With pulse shape analysis (PSA) the background in the HEIDELBERG-MOSCOW experiment around $\mathrm{Q}_{\beta \beta}$ is $4 \times 10^{-3}$ counts $/ \mathrm{kg} \mathrm{y} \mathrm{keV} \mathrm{[1,} \mathrm{which} \mathrm{is} \mathrm{a} \mathrm{factor} \mathrm{of} 4$ (5 referring to the line width) lower than that of GERDA with pulse shape analysis.

The amount of enriched material used in the GERDA measurement is $14.6 \mathrm{~kg}$, only a factor of 1.34 larger than that used in the HEIDELBERG-MOSCOW experiment. The background model is oversimplified and not yet adequate. It is not shown that the lines of their background can be identified. GERDA has to continue the measurement further $\sim 5$ years, until they can responsibly present an understood background. The present half life limit presented by GERDA of $\mathrm{T}_{1 / 2}^{0 \nu}>2.1 \times 10^{25} \mathrm{y}$ (90\% confidence level, i.e. $1.6 \sigma)$ is still lower than the half-life of $\mathrm{T}_{1 / 2}^{0 \nu}=2.23_{-0.31}^{+0.44} \times$ $10^{25} \mathrm{y}$ [1] determined in the HEIDELBERG-MOSCOW experiment.

PACS: 14.60.Pq; 23.40.-s; 29.40.-n; 95.55.Vj

Keywords: Neutrino mass and mixing; Double Beta Decay; HEIDELBERGMOSCOW experiment; High purity; Ge detectors; Majorana neutrino

*prof.klapdor-kleingrothaus@hotmail.de

†irinakv57@mail.ru 


\section{INTRODUCTION}

Nuclear double beta $(\beta \beta)$ decay is one of the flagships of non-accelerator particle physics searching for beyond standard model physics underground [2]. For many years (since 1992) the HEIDELBERG-MOSCOW experiment using the first enriched high-purity ${ }^{76} \mathrm{Ge}$ detectors dominates the field in sensitivity [3].

However, recently some fresh breeze arose in the field. The EXO and the Kamland-Zen experiments looking for $\beta \beta$ decay of ${ }^{136}$ Xe reached half-life limits of order of $10^{25}$ years (1.6 and $1.9 \times 10^{25}$ years (90\% c.l.), respectively) [4]. These results are consistent with HEIDELBERG-MOSCOW [1] within 1 or $2 \sigma$ with the matrix elements of [5]. They unfortunately suffer, however from low energy resolution ( $\sim 30$ times less than Ge detectors).

These days the GERDA experiment in Gran Sasso reported its first results [6, 7]. It used the idea of the GENIUS Project [8] namely installing naked Ge detectors in liquid nitrogen or liquid argon. Operating $14.6 \mathrm{~kg}$ of enriched ${ }^{76} \mathrm{Ge}$ - of them $10 \mathrm{~kg}$ from the HEIDELBERG-MOSCOW experiment - GERDA derived after exposure of $1.5 \mathrm{y}$ a lower limit of $\mathrm{T}_{1 / 2}^{0 \nu}>2.1 \times 10^{25} \mathrm{y}$ at $90 \%$ confidence limit $(1.6 \sigma)$ from their pulse-shape selected spectrum. On this basis they claim 'refuting of the HEIDELBERG-MOSCOW signal at high probability' [6]. To this experiment and this conclusion we have the following comments.

\section{GENERAL DEFICIENCIES OF THE EXPERIMENT AND THE ANALYSIS}

\subsection{HALF LIFE AND DOUBLE LINE}

The conclusion that the result of the HEIDELBERG-MOSCOW experiment (a signal at a $6.4 \sigma$ c.l. [1]) is refuted, is wrong.

The reason is very simple. The authors of [6, 7], compare their result to the line at (2038.1-2038.5) \pm 0.5 (stat.) \pm 1.2 (syst.) $\mathrm{keV}$ in the full (not PSA - treated) spectrum taken by HEIDELBERG-MOSCOW (exposure $71.7 \mathrm{~kg}$ ) see [9]. They ignore the result given in [1], that this line at $\mathrm{Q}_{\beta \beta}$ in the HEIDELBERG-MOSCOW experiment is a double line which cannot be resolved by the energy resolution of a Ge detector. Two lines of almost equal intensity occur at 2037.5 and $2039.3 \mathrm{keV}$. They can be separated, however, by pulse shape analysis (PSA), since the first one consists essentially of single site events as expected for a $0 \nu \beta \beta$ line, the second essentially of multiple site events, as expected for a gamma line. Their intensities are $11.0 \pm 1.8$ and $10.3 \pm 3.3$, respectively, adding to the line found in the full (not PSA-treated) spectrum of $19.6 \pm 5.4$ events obtained after $51.39 \mathrm{~kg} \mathrm{y}$ (the time during which the time structure of pulses has been recorded in the HEIDELBERG-MOSCOW experiment). The $0 \nu \beta \beta$ half life is consequently $\left(2.23_{-0.31}^{+0.44}\right) \times 10^{25}$ years [1] and not $\left(1.19_{-0.22}^{+0.38}\right) \times 10^{25} \mathrm{y}$ as deduced in [9] from the full spectrum and as assumed in the GERDA report.

Therefore, in their window around $\mathrm{Q}_{\beta \beta}$ GERDA should expect 3.1 10.8 events only (but not $5.9 \pm 1.4$ ( $1 \sigma$ error!) as they claim). This signal should be searched at an energy of $2037.5 \mathrm{keV} \pm 0.5$ (stat.) \pm 1.2 (syst.), where the single site line is found, not at $2039 \mathrm{keV}$ where we observe the multiple $\gamma$-line. 
The fact that the line at $\mathrm{Q}_{\beta \beta}$ in the full spectrum in the HEIDELBERG-MOSCOW experiment is a double line, has been observed independently also by I. Kirpichnikov [10]. He found further indication of a tiny third line at $\sim 2034.5 \mathrm{keV}$, unresolved from the line seen at $2038.5 \mathrm{keV}$. It shows up also in our spectrum of multiple site events rejected by PSA (see Fig.5 and Fig.6 of DARK2007 Proc. [1, 2]). He claimed that the lines at $2039.3 \mathrm{keV}$ and $2034.5 \mathrm{keV}$ each are a sum line of two consecutive gamma transitions (as it is the case also for the $2016.7 \mathrm{keV}$ line - see below and [12]). He also showed that this is supported by the GEMMA Experiment [11]. So their existence does not contradict to the findings of Gromov et. al [13] and Dörr et al. [14] that there is no gamma transition of this energy in known radioactive isotopes.

Concluding GERDA has to compare its $1.6 \sigma$ limit of $2.1 \times 10^{25} \mathrm{y}$ to the HEIDELBERG-MOSCOW $6.4 \sigma$ signal of single site events yielding $\mathrm{T}_{1 / 2}=\left(2.23_{-0.31}^{+0.44}\right) \times 10^{25} \mathrm{y}[1]$. This means that the GERDA limit is lower than the HEIDELBERG-MOSCOW half life, and is fully consistent with HEIDELBERG-MOSCOW, but because of its low statistics GERDA cannot proof anything at this moment.

In their slides 54-57 [6] they show their 90\% upper limit. This is not a fit, but simple superposition of a Gaussian line with fixed energy, intensity and width on the background. In the upper parts of slides 56, 57 [6] they compare this to their expectations from the erroneously assumed half life for the HEIDELBERG-MOSCOW experiment [9] - and not at the energy position where the line has been observed. The correct expectation from HEIDELBERG-MOSCOW [1] is shown in Fig.1 of this paper, which should replace the

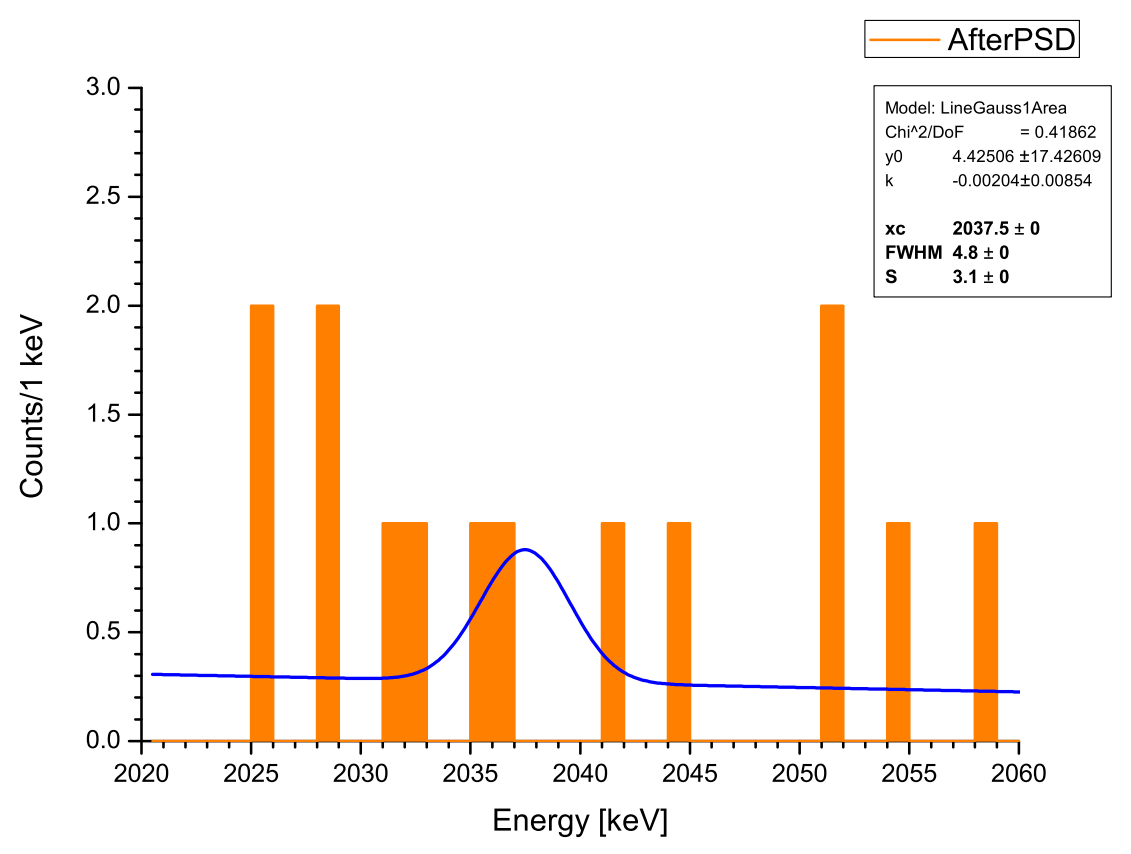

Figure 1: Gerda spectrum after pulse shape discrimination (PSD), from [6, 7]. The solid line corresponds to the correct expectation from the HEIDELBERG-MOSCOW experiment [1, 2] (see text) 
dotted red line in the slides 56, 57 of [6]. In Fig. 1 from the 5 parameters of the fit program all, except the two background parameters, are fixed: position of the line and its expected intensity according to [1, FWHM according to GERDA experimental resolution.

No contradiction to the GERDA result can be seen here.

But even the discrepancy between the limit of $\mathrm{T}_{1 / 2}>2.1 \times 10^{25} \mathrm{y}(90 \%$ c.l., i.e. $1.6 \sigma$ level) of GERDA and the half-life of $1.19 \times 10^{25} \mathrm{y}$, which they assumed erroneously, is less than the $2 \sigma$ uncertainty of this half life (which is $\left(1.19_{-0.39}^{+1.08} \times 10^{25} \mathrm{y}\right)$. Already this certainly would not justify the strong statement of refuting the HEIDELBERG-MOSCOW result.

\subsection{BACKGROUND AND BACKGROUND MODEL BUILDING}

It has been mentioned that the main goal of reaching a background of $10^{-2}$ counts $/ \mathrm{kg} y \mathrm{keV}$, or by an order of magnitude lower than in the HEIDELBERG-MOSCOW experiment, was not reached by GERDA. The background of GERDA in the energy window 2000-2060 keV around $\mathrm{Q}_{\beta \beta}$ is 0.031 counts $/ \mathrm{kg}$ y keV. This is a factor of 3.5 lower than the HEIDELBERGMOSCOW background of $0.113 \pm 0.007$ counts $/ \mathrm{kg}$ y keV [9]. It is, however, only by a factor of 2.3 smaller than HEIDELBERG-MOSCOW if we refer to the line width, i.e. in units of counts $/ \mathrm{kg} y$ energy resolution. It is the latter value which defines the sensitivity of the experiment concerning background (without PSA). With pulse shape analysis the background in the HEIDELBERG-MOSCOW experiment is $\sim 4 \times 10^{-3}$ counts $/ \mathrm{kg}$ y keV [1] around $\mathrm{Q}_{\beta \beta}$ (range 2000-2060 $\mathrm{keV}$ ). This is a factor of 4 (5, when referring to the line width) lower than found by GERDA with PSA.

A general criticism has to be made concerning the treatment of background by GERDA, which is completely unsufficient at this moment. The main point is that they do not show that all lines in the spectrum are understood. The spectra are shown in [6, 7] - except for a range of $\sim 1900-2200 \mathrm{keV}$ around $\mathrm{Q}_{\beta \beta}-$ only binned into energy bins of $5 \mathrm{keV}$. This is hardly adequate to an energy resolution of $4.8 \mathrm{keV}$. More critical, is that they compare most part of their spectra in a $30 \mathrm{keV}$ binning to a background model averaging also over $30 \mathrm{keV}$. This means that for most part of the spectrum individual gamma lines are not shown and their intensities were not determined or at least are not listed. In this way the usual procedure of localizing the sources of radioactive impurities in the setup cannot be applied. Further, in such way it cannot be checked whether there exist lines in the spectrum not included in their background model. Strange is also, that they (their slide 55, from [6] ) exclude 'lines' at 2104 and $2119 \mathrm{keV}$ from their background - at least the first of them being not visible. If the 2104 and $2119 \mathrm{keV}$ lines are accepted as lines than there are $m$ a $n$ y lines in their $2 \mathrm{keV}$-binned spectrum to be accepted and to be explained.

Also about the background in the $i n d i v i d u$ a $l$ detectors nothing can be said in this way. It is clear that under such circumstances the background cannot yet be claimed to be understood.

In the HEIDELBERG-MOSCOW experiment more than 70 lines have been seen and identified in the spectrum [14, 13]. A special investigation of the background around $\mathrm{Q}_{\beta \beta}$, and of the intensity ratios of the ${ }^{214} \mathrm{Bi}$ lines, some of which occur in the window around $\mathrm{Q}_{\beta \beta}$ (range $2000-2100 \mathrm{keV}$ ), has been performed with a ${ }^{226} \mathrm{Ra}$ source [12]. In particular the effect of true coincidence summing on the intensities has been studied, in particular, for the line at $2016.7 \mathrm{keV}$, which as E0 transition can be seen only as sum line of two 
consecutive gamma transitions - as the line identified at $2039.3 \mathrm{keV}$ (see above).

Concerning their background models: The comparison between measured spectrum and calculated background in the GERDA reports, in spite of the smoothening of the data in $30 \mathrm{keV}$ bins - which suppresses local deviations - shows differences up to a factor of 2 and more (a factor of 2.3 in the region around $\mathrm{Q}_{\beta \beta}$ !) (Slides 33, 34 in [6]). This raises the question whether these models are sufficient.

Highly surprising is the statement that a socalled minimal background model not including lines from ${ }^{214} \mathrm{Bi}$ should be sufficient. ${ }^{214} \mathrm{Bi}$ was found in the HEIDELBERGMOSCOW experiment to yield the famous lines closest to $\mathrm{Q}_{\beta \beta}$ [9, 12, and is clearly seen also by GERDA (e.g. at $2204 \mathrm{keV}$ ), and seems to show up also already in the region around $\mathrm{Q}_{\beta \beta}$.

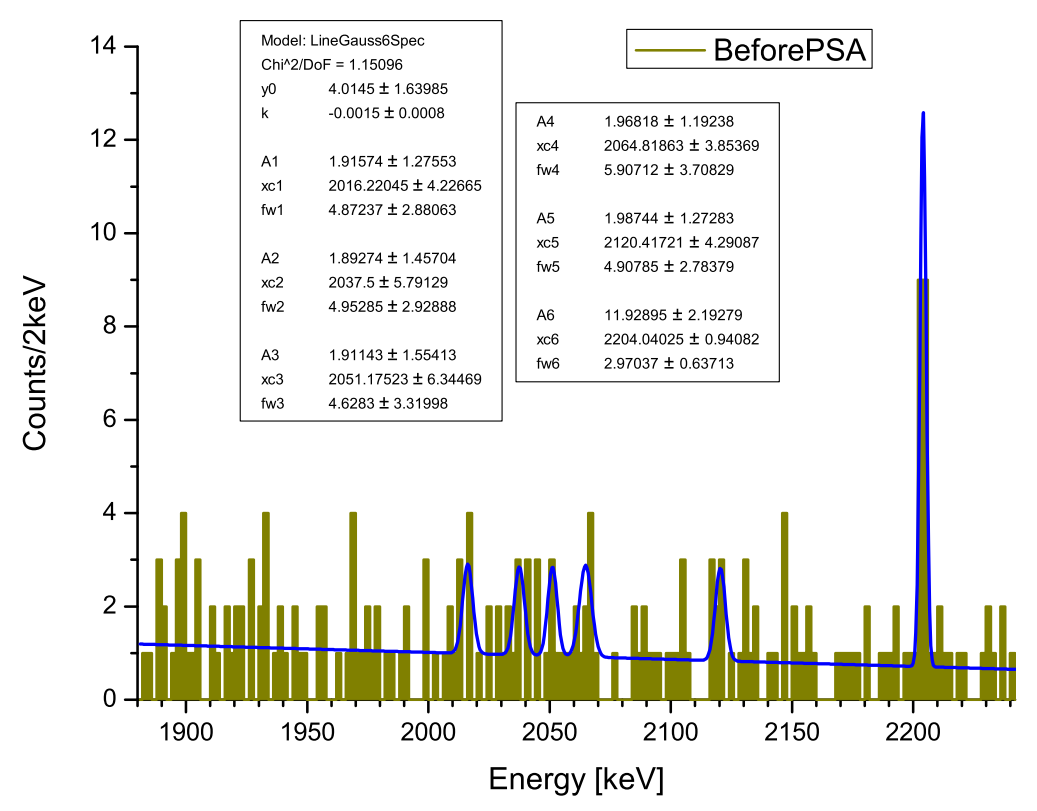

Figure 2: Gerda spectrum before PSD with $2 \mathrm{keV}$ - binning (from [6, 7]). Besides the strong ${ }^{214} \mathrm{Bi}$ line at $2204 \mathrm{keV}$, our fit finds indications of lines (on 1-2 $\sigma$ level) at known positions of ${ }^{214} \mathrm{Bi}$ lines $2016.7 \mathrm{keV}$, (not separable from 2010.7 and $2021.8 \mathrm{keV}$ ), $2052.9 \mathrm{keV}$, $2119 \mathrm{keV}$. Further it finds a line at $2037.5 \mathrm{keV}$ (not separable from lines 2034.5 and $2039.3 \mathrm{keV}$ ). (The parameter 'A' of the fit is connected with the intensity ' $\mathrm{N}$ ' by $\mathrm{N}=\mathrm{S} / 2$, $\mathrm{S}=\mathrm{A} \times(\mathrm{FWHM} / 2) \times \sqrt{\pi / \ln 2}$. The other parameters are self-explainable (see text))

Fig. 2 shows a fit which could complement their full spectra shown in slides 54,55 , 56 (lower part) of [6].

Besides the strong ${ }^{214} \mathrm{Bi}$ line at $2204 \mathrm{keV}$, some lines of low statistics (between 1 and $2 \sigma)$ are indicated in the range around $\mathrm{Q}_{\beta \beta}(2000-2120 \mathrm{keV})$ at energies corresponding to ${ }^{214} \mathrm{Bi}$ lines (2016.7 keV, not resolvable from 2010.7 and $2021.9 \mathrm{keV}$ Bi lines), $2052.9 \mathrm{keV}$, $2119 \mathrm{keV}$. A line at $2065 \mathrm{keV}$ is not understood. Also a line at $2037.5 \mathrm{keV}$ is indicated, not separable from 2034.5 and $2039.3 \mathrm{keV}$ lines. Its intensity of $4.9 \pm 3.8$ counts is consistent with the expectation from HEIDELBERG-MOSCOW [1, 2], which is $(4.4 \pm 1.0)$ 
counts, adding the expectations from the $2037.5 \mathrm{keV}$ line and for half of the $2039.3 \mathrm{keV}$ line (because of lower background of GERDA without PSD). In case GERDA would not see the $2039.3 \mathrm{keV}$ line, the expected value from HEIDELBERG-MOSCOW would be $3.1 \pm 0.8$, both being fully consistent with the above given fit. Considering the number of events to be expected in the GERDA spectrum before PSD, in a region of $2 \mathrm{FWHM}$ $(4.6 \sigma)$ around $2037.5 \mathrm{keV}$, a value of $8.9 \pm 1.8$ events is expexted,with the background of 0.5 counts per keV determined by the fit in Fig.2. The observed value of events in this range is 10 . So the present GERDA result is fully consistent with the result of HEIDELBERG-MOSCOW [1, 2].

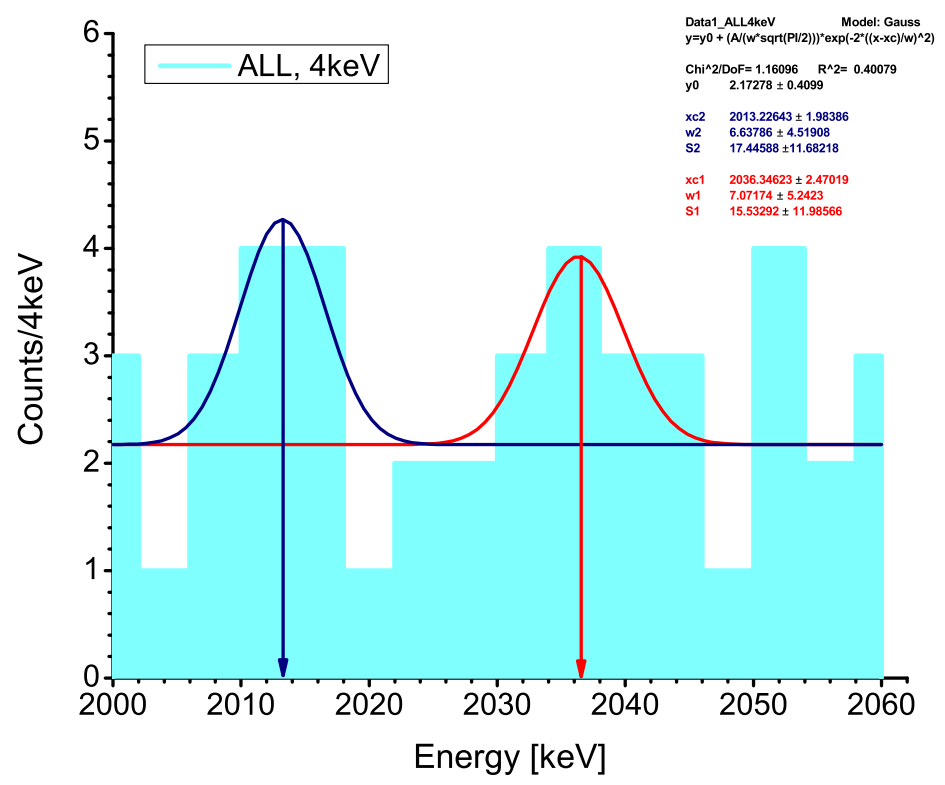

Figure 3: The Gerda spectrum before PSD, with $4 \mathrm{keV}$ - binning and our fit. Two structures arise around $2014 \mathrm{keV}$ (unresolved ${ }^{212} \mathrm{Bi}-2010.7,2016.7,2021.8 \mathrm{keV}$ ), and a broad unresolved structure covering the range 2034-2040 keV (lines 2034.5, 2037.5, 2039.3 keV). (In the fit ' $\mathrm{S}$ ' determines the number of counts $\mathrm{N}$ in the line, $\mathrm{N}=\mathrm{S} / 4$ )

Fig. 3 shows the full GERDA spectrum (before PSA) with a binning of $4 \mathrm{keV}$. Two structures dominate the spectrum, a broad line at the location of the unresolved ${ }^{214} \mathrm{Bi}$ lines at 2010.7, 2016.7 and $2021.8 \mathrm{keV}$ (known from the HEIDELBERG-MOSCOW experiment) [9], and a broad unresolved structure covering the range 2034 to $2040 \mathrm{keV}$, which includes the lines 2034.5, 2037.5 and $2039.3 \mathrm{keV}$ known from HEIDELBERG-MOSCOW [1]. The fit yields for the second line $\mathrm{E}=2036.4 \pm 2.5 \mathrm{keV}$ and an intensity of $3.9 \pm 3.0$ events, consistent with the expectation (see above) of $4.4 \pm 1.0$ events. A word of caution: It should be mentioned, however, that different ways of $4 \mathrm{keV}$ binning can give rather different result. Thus Figs. 2 and 3 show, that at the low statistics GERDA has at present, and with the low energy resolution the GERDA detectors have, at this moment it is premature and marginal to search for resolved lines in this region. The statistics of GERDA at present is simply not sufficient to check the result of the HEIDELBERG-MOSCOW experiment. But 
what is found, is consistent with the expectation from HEIDELBERG-MOSCOW 1, 2].

The GERDA report claims no excess of signal counts above background. However, even according to their Table (Slide 52 in [6]) an excess is there.

Unusual is that the authors show and determine the background spectrum not over the full measuring time (November 2011 until May 2013) but only until January 2013 [6, 7, 18. The reason should be given: Why $4-5$ months of statistics remained unused? One would need further some proof that the background during the period January to May 2013 was the same as in the period before!

\subsection{DETECTOR RESOLUTION AND STABILITY OF ELECTRONICS}

The energy resolution and its time stability of the coaxial detectors through 1.5 years of operation is rather modest, the energy of the $2614.5 \mathrm{keV}$ Th line floating between -1.4 and $+2.5 \mathrm{keV}$ around the average value. The average resolution lies for the different detectors between 4.2 and $5.8 \mathrm{keV}$, and averaged over the detectors is $4.8 \mathrm{keV}$. This is a factor of 1.5 worse than the resolution of the same detectors during eight years of measurement in the HEIDELBERG-MOSCOW experiment, which was $3.27 \mathrm{keV}$ [9]. The reason should be given. This could also indicate some time instability of the electronics in the GERDA experiment. No analysis of a possible temperature dependence of the setup and electronics is mentioned.

\subsection{PULSE SHAPE ANALYSIS}

The training of the neuronal net for GERDA is done with the method given in [16] using the $1592.5 \mathrm{keV}$ double escape line of the $2614.5 \mathrm{keV}{ }^{228} \mathrm{Th}$ line for simulating single site events, and the $1612 \mathrm{keV}$ total absorption peak from the ${ }^{228} \mathrm{Th}$ daughter nuclide ${ }^{212} \mathrm{Bi}$ for multiple site events. Unfortunately the time structure of all individual events in the relevant range of energy around $\mathrm{Q}_{\beta \beta}$ is not shown by GERDA. The reduction of the $\gamma$-background is rather modest (order of factor 2).

There are, however, still differences between $0 \nu \beta \beta$ (and $2 \nu \beta \beta$ ) events and DE- $\gamma$-events in time structure and size (partial volumes in the Ge detector inside which the energy of the events is released), see Monte Carlo simulations in [15].

These differences of $0 \nu \beta \beta$ events of different effective neutrino mass $m$ and righthanded current parameters $\theta, \lambda$ from single site (DE) $\gamma$ events are such, that even with the typical spatial resolution of a large Ge detector it might not be excluded to separate $0 \nu \beta \beta$ events sharper from any kind of $\gamma$-event, if the neuronal net could be properly 'calibrated'.

In [1] this has been tried in some empirical way, with the result of a drastic further reduction of the whole $\gamma$-background to $\sim 4 \times 10^{-3}$ counts $/ \mathrm{kg}$ y keV (see [1] and also Fig.33 (left) in [9]). With this reduction the candidate $0 \nu \beta \beta$ line stands out clearly of the background. 


\subsection{PROBLEMS WITH DETECTORS IN LIQUID ARGON?}

Despite GERDA operated its detectors in liquid argon in shrouds of very thin copper (does this mean that it is tried to not use naked detectors?) already two detectors could not be used. This led to the fact that instead of $17.7 \mathrm{~kg}$ of enriched material only $14.6 \mathrm{~kg}$ have been used (not much more than the $10.9 \mathrm{~kg}$ used in the HEIDELBERG-MOSCOW experiment). As reason was given too high leakage current. Nothing is said in the report about the behaviour of the leakage currents of the other detectors as function of running time in the liquid argon.

The experience from our GENIUS Test Facility, in which we operated six (nonenriched) naked Ge detectors over the period of three years in Gran Sasso was the following [19, 20]: Limited long-term stability of naked detectors in liquid nitrogen as a result of increasing leakage current. After three years none of the six detectors was working any more with the nominal leakage current. Three of the detectors did not work any more at all.

\section{CONCLUSION}

The most sensitive double beta decay experiments at present under operation, EXO and KAMLAND-ZEN [4] and GERDA [6, 7] reported lower limits for neutrinoless double beta decay on a $90 \%$ c.l., which are consistent with the $6.4 \sigma$ signal delivered by the HEIDELBERG-MOSCOW Experiment [1]. All of these experiments plan improvements of their sensitivity. The future $\mathrm{SNO}+$ experiment with the $\beta \beta$ emitter ${ }^{130} \mathrm{Te}$ still is only under discussion [17]. It is obvious, that it will take quite some more years, until checking of the HEIDELBERG-MOSCOW positive result will become possible.

In the case of EXO and KAMLAND-ZEN the modest energy resolution of $\sim 90 \mathrm{keV}$ may make serious problems in the moment where indications of a signal might be found - remember the lines close to the $0 \nu \beta \beta$ line from HEIDELBERG-MOSCOW .

In the case of GERDA:

1. should be improved considerably the treatment of background data, which at present is on an unacceptable level. It is not acceptable that no list of identified lines and intensities exists and consequently no comparison with expectations. Consequently no satisfactory localisation of the radioactive impurities in the setup could be performed. It is further not acceptable that lines remain unexplained in the spectrum.

2. the statistics of the experiment has to be decisively improved before any relevant statements can be made, to avoid premature conclusions as in the present report.

3. the reasons for the limited energy resolution of the detectors have to be explained, and the resolution has to be improved to an acceptable level.

4. the time structure of their events in the relevant energy range around $\mathrm{Q}_{\beta \beta}$ should be individually shown, and also their fits by their pulse shape approximation library (as has been done in [1]).

5. In view of the experience with GENIUS-TF [19, 20] the development of the leakage currents of the detectors as function of time should be shown. After two detectors 
already did not work because of too high leakage current in the present run of GERDA, it should be made sure that not more detectors will be lost by the operation in liquid argon.

Because of its similar background and detector mass GERDA would require similar measuring times as HEIDELBERG-MOSCOW to get comparable statistics.

Some outlook on the future of $\beta \beta$ experiments is given in [20].

\section{ACKNOWLEDGEMENTS}

The authors gratefully acknowledge the important contribution of Dr. S.N. Karpov to this paper. It is our pleasure to give our deeply felt thanks here to all friends and colleagues, who have supported us so efficiently in various ways on our way through double beta decay research during the last twenty-five years.

\section{References}

[1] Klapdor-Kleingrothaus H.V., Krivosheina I.V. The Evidence for the Observation of $0 \nu \beta \beta$ Decay: The Identification of $0 \nu \beta \beta$ Events from the Full Spectra// Mod. Phys. Lett. A. 2006. V.21. P. 1547-1566;

Klapdor-Kleingrothaus H.V. Hot Dark Matter and Neutrinoless Double Beta Decay: World Status of the Field // Proc. of $<<$ DARK 2007 $>>$, Int. Conf. on Dark Matter in Astroparticle and Particle Physics, Sydney. Australia. 24.-28. Sep. 2007. eds. Klapdor-Kleingrothaus H.V. and Lewis G. L., World Scientific. Singapore. 2008. PP. 442-467 (reprinted also in [2]).

[2] Klapdor-Kleingrothaus H.V. Seventy Years of Double Beta Decay - From Nuclear Physics to Beyond-Standard-Model Particle Physics// World Scientific, Singapore, 2010. 1520 pages; htpp://www.worldscientific.com/worldscibooks/10.1142/6921

[3] Happy Birthday, Particle Hunter // CERN Courier. March. 2012. P. 34; Team Reports Neutrinoless Double Beta Decay //CERN Courier. March. 2002. P. 5.

[4] Auger M. et al. (EXO collab.) Search for Neutrinoless Double-Beta Decay in ${ }^{136} \mathrm{Xe}$ with EXO-200 // Phys. Rev. Lett. 2012. V.109. P.032505. arXiv:1205.5608[hep-ex]; Gando A. et al. (KAMLAND-ZEN collab.) Measurement of the Double-Beta Decay Half-Life of Xe-136 with the KamLAND-Zen Experiment// Phys. Rev. 2012. V.C85. P. 045504. arXiv:1201.4664[hep-ex].

[5] Staudt A., Muto K., Klapdor-Kleingrothaus H.V. Calculation of Two Neutrino and Zero Neutrino Double Beta Decay Rates// Europhys. Lett. 1990. V.13 PP. 31-36.

[6] Schönert S. GERDA Presents the First Results on Neutrinoless Double Beta Decay of 76Ge from Phase I// Seminar at Gran Sasso Underground Laboratory. 16. July. 2013. http://streaming.lngs.infn.it

[7] Cattadori C.M. et al. First GERDA Results on $0 \nu \beta \beta$ of ${ }^{76} \mathrm{Ge} / /$

EPS-HEP Conf. 17.-24. July. 2013. Stockholm, https://indico.cern.ch/conferenceOtherViews.py?confId=218030\&view=standard 
[8] Klapdor-Kleingrothaus H.V., Hellmig J., Hirsch M. Future Perspectives of Double Beta Decay and Dark Matter Search - GENIUS// J. Phys. G: Nucl. Part. Phys. 1998. V. 24. PP. 483-516. and

'Naked' Crystals go Underground// CERN Courier. July/August. 2003. V. 43 N. 6 P. 9 and e-Print: hep-ph/0307329.

[9] Klapdor-Kleingrothaus H.V., Krivosheina I.V., Dietz A. et al. Search for Neutrinoless Double Beta Decay With Enriched Ge-76 in Gran Sasso 1990-2003// Phys. Lett. 2004. V. B586. PP. 198-212 and e-Print: hep-ph/0404088;

Klapdor-Kleingrothaus H.V., A. Dietz, I.V. Krivosheina et al. Data Acquisition and Analysis of the Ge-76 Double Beta Experiment in Gran Sasso 1990-2003// Nucl. Instr. and Meth. 2004. V. A522. PP. 371-406 and e-Print: hep-ph/0403018.

[10] Kirpichnikov I.V. Klapdor's Claim for the Observation of the Neutrinoless Double Beta-Decay of Ge-76. Analysis and Corrections.// 2010. arXiv:1006.2025[hep-ph].

[11] Beda A.G., Brudanin V.B. et al. Status of the Experiment on the Measurement of the Neutrino Magnetic Moment with the Spectrometer GEMMA// Phys. At. Nuclei. 2004. V. 67. PP. 1948-1952; Yad. Fizika. 2004. V. 67. PP. 1973-1976.

[12] Klapdor-Kleingrothaus H.V., Chkvorets O., Krivosheina I.V., Tomei C. Measurement of the Bi-214 Spectrum in the Energy Region Around the Q Value of Ge-76 Neutrinoless Double Beta Decay// Nucl. Instr. Meth. 2003. V.A511. PP. 335-340 and e-Print: hep-ph/0309157;

Klapdor-Kleingrothaus, H.V., Dietz A., Krivosheina I.V. et al. Background Analysis Around $\mathrm{Q}_{\beta \beta}$ for ${ }^{76} \mathrm{Ge}$ Double Beta Decay Experiments, and Statistics at Low Count Rates// Nucl. Instrum. Meth. 2003. V. A510 PP. 281-289 and e-Print: hep-ph/0308275; and Support of Evidence for Neutrinoless Double Beta Decay// Phys. Lett. 2004. V. B578 PP. 54-62 and e-Print: hep-ph/0312171.

[13] Gromov K.Ya., Bednyakov V.A., Fominykh V.I. et al. Background Structure in the Heidelberg-Moscow Experiment on the Search and Investigation of Double Beta Decay of Ge-76// J. Part. Nucl. Lett. 2006. V. 3. PP. 157-164.

[14] Dörr C., Klapdor-Kleingrothaus H.V. New Monte-Carlo Simulation of the HEIDELBERG-MOSCOW Double Beta Decay Experiment// Nucl. Instr. Meth. 2003. V. A513. PP. 596-621.

[15] Klapdor-Kleingrothaus H.V., Krivosheina I.V., Titkova I.V. Particle and Nuclear Physics Parameters: How do They Affect the Tracks of Double Beta Events in a Germanium Detector, and Their Separation from Gamma Events// Phys. Lett. 2006. V. B632. PP.623-631;

Theoretical Investigation of the Dependence of Double Beta Decay Tracks in a Ge Detector on Particle and Nuclear Physics Parameters and Separation from Gamma Ray Events// Phys. Rev. 2006. V. D73. P. 013010;

Klapdor-Kleingrothaus H.V., Krivosheina I.V., Mironov V. and Titkova I.V. Microscopic Calculations of Signals of Double Beta Decay in a Ge-76 Detector and First Application to the Heidelberg-Moscow Experiment// Phys. Lett. 2006. V. B636. PP. 235-247. 
[16] Majorovits B. and Klapdor-Kleingrothaus H.V. Digital Pulse Shape Analysis by Neural Networks for the Heidelberg-Moscow Double Beta-Decay Experiment// Eur. Phys. J. 1999. V. A6. PP. 463-469 and e-Print: hep-ex/9911001.

[17] Mottram M. et al. SNO+// EPS-HEP Conf. 17.-24. July. 2013. Stockholm https://indico.cern.ch/conferenceOtherViews.py?confId=218030\&view=standard.

[18] Knöpfle K.T.// priv. communication July. 23. 2013.

[19] Klapdor-Kleingrothaus H.V., Tomei C., Krivosheina I.V., et al. First 10-kg of Naked Germanium Detectors in Liquid Nitrogen Installed in the GENIUS Test Facility// Nucl. Instr. Meth. 2003. V.A511. PP. 341-346 and e-Print: hep-ph/0309170;

The GENIUS-Test-Facility: First Results on Background from Rn-222 Daughters// Nucl. Instr. Meth. 2004. V. A530. PP. 410-418;

Status of GENIUS-TF-II and TF-III: The Long-Term Stability of Naked Detectors in Liquid Nitrogen// Nucl. Instr. Meth. 2006. V. A566. PP. 472-476.

[20] Klapdor-Kleingrothaus H.V. Lessons After the Evidence for 0 $\nu$ Beta Beta Decay// Phys. Scripta. 2006. V. T127 PP.40-42;

Klapdor-Kleingrothaus H.V., Krivosheina I.V. Lessons After 3 Years of Running GENIUS-TF in Gran Sasso// Phys. Scripta. 2006. V. T127 PP. 52-53;

Klapdor-Kleingrothaus H. V. Lessons After the Evidence for Neutrinoless Double Beta Decay: The Next Step Int. J. Mod. Phys. 2008. V. E17 PP. 505-517. 